\title{
INFRARED MICROSPECTROSCOPY OF DIAMOND IN RELATION TO MANTLE PROCESSES.
}

\author{
Mendelssohn ${ }^{(1)}$, M.J.; Milledge ${ }^{(1)}$, H.J.; Cooper ${ }^{(1),}{ }^{(2)}$, G.I. and Meyer ${ }^{(3)}$, H.O.A.
}

(1) Crystall. \& Min. Phys. Unit, Dept. Geol. Sci., University College London, Gower Street, London WC1E 6BT, U.K; (2) Physics Department, University of York, Heslington, York YO1 IUY, U.K.; (3) Dept. Earth \& Atmos. Sci., Purdue University, West Lafayette, IN 47907 USA.

Infrared spectra of diamond reveal the presence of broad absorption bands in the onephonon region $\left(1332 \mathrm{~cm}^{-1}\right.$ to $\left.900 \mathrm{~cm}^{-1}\right)$ which vary substantially within and between specimens in both structure and magnitude, although diamond, being centrosymmetric, should not exhibit one-phonon absorption at all. These bands are therefore associated with defects of some kind, generally assumed to consist of nitrogen in various states of aggregation ranging from single nitrogen atoms (Type Ib) present in synthetic diamonds through 2nitrogen (Type IaA) and 4-nitrogen (Type IaB) aggregates to extend defects of variable size (platelets) associated with a relatively narrow absorption band near $1368 \mathrm{~cm}^{-1}$. Although this band is outside the 1-phonon region, its development appears to be well correlated with the development of the IaB spectrum in many diamonds, but HP/HT laboratory experiments have shown that it can be reduced or destroyed without affecting the IaB spectrum.

Laboratory experiments have also shown that whereas the activation energy for the Ib -- IaA defect aggregation is low enough for the process to be complete in most natural diamonds, the activation energy for the IaA -- [IaB + Platelet $]$ aggregation is high enough for the process to be incomplete in most natural diamonds. An experimental determination of this activation energy can therefore be used to estimate the residence times and temperatures associated with separate growth horizons in diamonds from different localities or within a particular locality.

Our $\mathrm{HP} / \mathrm{HT}$ experiments indicate an activation energy of $7 \mathrm{EV}$, and show conclusively that the aggregation is a second-order process, i.e. it is concentration dependent. However, any given aggregation state is a product of temperature and time, and cannot define either independently, but if the temperature can be established by, for example, inclusion geothermometry, then the infrared spectrum can imply a residence time.

If infrared spectroscopy can provide reliable time/temperature information for individual diamonds, the fact that it is a rapid and non-destructive technique will permit studies of the relative homogeneity or otherwise of diamond populations to be made on statistically significant numbers of specimens more rapidly than is possible from studies of inclusion geochemistry, and importantly, for specimens which do not contain inclusions.

Our results confirm earlier observations that the platelet peak can be destroyed in such HP/HT experiments, but the morphological changes produced by the HP/HT graphitization differ from those reported previously. 
Table 1. A comparison between the temperatures calculated using mineral inclusion geothermometers and those calculated using the ' $\mathrm{A}$ ' $\rightarrow$ ' $\mathrm{B}$ ' aggregation state, found from the one phonon region of the diamond infrared spectrum.

DIAMOND TEMP ${ }^{\circ} \mathrm{C}(\mathrm{M} . \mathrm{L}) \mathrm{TEMP}^{\circ} \mathrm{C}(\mathrm{R} \cdot \mathrm{R} . \mathrm{a})$ TEMP ${ }^{\circ} \mathrm{C}(\mathrm{C} \cdot \mathrm{R}, \mathrm{b})$

$\begin{array}{llll}\text { RSOV02 } & 1292 & 1017 \rightarrow 1043 & 1161 \rightarrow 1192 \\ \text { RSOV04 } & 910 \rightarrow 970 & 1079 \rightarrow 1170 & 1233 \rightarrow 1337 \\ \text { TP9 } & 1202 \rightarrow 1295 & 1066 \rightarrow 1110 & 1217 \rightarrow 1269\end{array}$

M.I. The temperature calculated using inclusion geothermometry.

I.R. The temperature calculated using the infra-red spectrum. A storage time of between 1 and 2 Gy was assumed for the Romaria (Brazil) diamonds (RSOW) and of between 1.5 and $3 \mathrm{~Gy}$ for TP9.

a) Using Activation Energy for diamond $=674.8 \mathrm{KJmol}^{-1}$ (This work)

b) Using Activation Energy for diamond $=733.0 \mathrm{KJmol}^{-1}$ (Evans and Qi 1982)

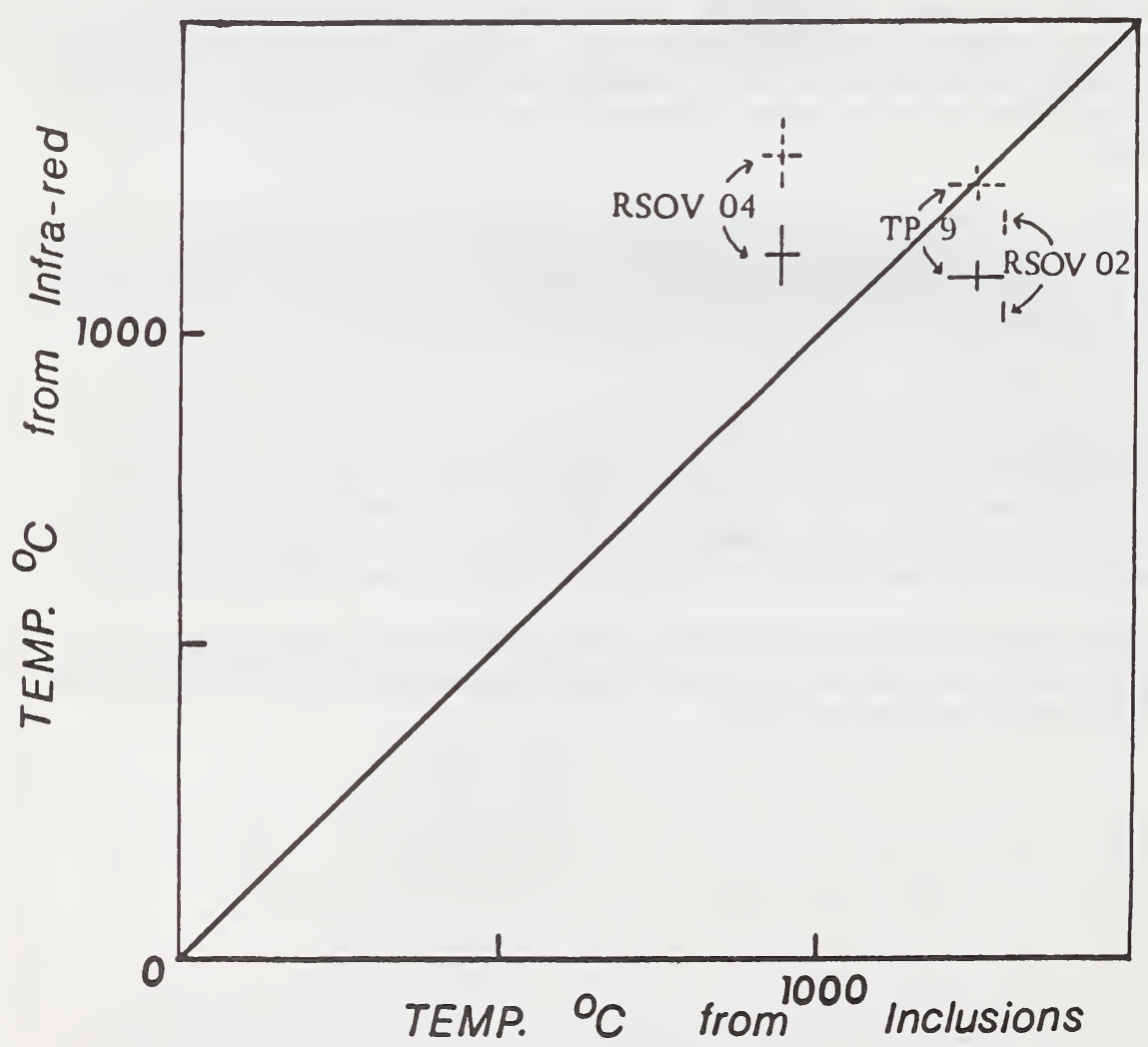

Figure 1. A comparison between the temperatures calculated using mineral inclusion geothermometers and those calculated using the ' $A$ ' $\rightarrow$ ' $B$ ' aggregation state found from the one-phonon region of the diamond infra-red spectrum.

- results using Activation Energy for diamond $=674.8 \mathrm{KJmol}^{-1}$ (chapter 4 )

..- results using Activation Energy for diamond $=733.0 \mathrm{KJmol}^{-1}$ (Evans and Qi 1982) 